\title{
Gedanken zum Weltagrarbericht aus historisch-ökologischer Sicht
}

\section{Die Qualität des International Assessment of Agricultural Knowledge, Science and Technology for Development müssen Landwirtschafts- expert(inn)en beurteilen. Aus Sicht der Historischen Ökologie stellt sich die grundsätzliche Frage, ob ein System wie}

die globale Landwirtschaft überhaupt einem geordneten, zielgerichteten Planungsprozess zugänglich ist. Erfahrungen aus der Vergangenheit geben Anlass zu Zweifeln.
Matthias Bürgi

Reflections Upon the IAASTD by a Historical Ecologist | GAIA 17/3 (2008): 293-294

Keywords: agricultural history, driving forces, historical ecology, IAASTD, land use, planning

D ie Anfrage, ob ich einen Beitrag zum Agassessment ${ }^{1}$ verfassen könne, überraschte mich, war ich doch dem Begriff noch nie begegnet. Um wenigstens eine qualifizierte Absage schreiben zu können, verschaffte ich mir einen raschen Überblick. Eine Internetsuche führte mich zu offiziellen Dokumenten, Protokollen und Vortragstexten. ${ }^{2}$ Sie zeigten das Bild einer breit abgestützten, umfassenden, internationalen Studie zum Zustand der Landwirtschaft, der durch Nichtregierungsorganisationen aktiv und kritisch begleitet wird.

Mein Fachgebiet ist die Historische Ökologie, das heißt, mich interessieren ökologische Aspekte der Mensch-Umwelt-Interaktionen im Laufe der Zeit. Viele der im Agassessment aufgeworfenen Fragen erfordern die Berücksichtigung historisch-ökologischer Aspekte. Das weckte mein Interesse, und ich beschloss, mir einige Gedanken dazu zu machen.

Worum geht es eigentlich im Agassessment? Die Aufgabe ist klar deklariert: „an international assessment of the role of agricultural knowledge, science and technology (AKST) in reducing hunger and poverty, improving rural livelihoods and facilitating equitable, environmentally, socially and economically sustainable development“ (IAASTD 2008, S. 3).

„Recht ambitioniert!“, dachte ich - wobei mir das mit diesem Gedanken einhergehende Schmunzeln eingedenk der Ehrenhaftigkeit dieser Zielsetzung und angesichts der Liste der betei-

1 Offiziell heißt das Projekt International Assessment of Agricultural Knowledge, Science and Technology for Development (IAASTD), im Deutschen Weltagrarbericht. Der vorliegende Artikel verwendet, abgeleitet aus der Internetadresse www. agassessment.org, diese "handlichere" Bezeichnung.

2 Die meisten dieser Unterlagen waren vorläufig und wurden später bis zur endgültigen Veröffentlichung, die im Herbst 2008 erwartet wird, wieder von der Homepage genommen. ligten Expert(inn)en sofort wieder von den Lippen wich. Was genau verführt eigentlich angesichts hehrer Ziele zum Schmunzeln? Ist es die Spannung zwischen den Zielen und den effektiven Einflussmöglichkeiten? Vermutet der bösartige Schmunzler naive Selbstüberschätzung der Zielsetzenden?

Eine relevantere Frage lautet: Welche Triebkräfte führen zu dieser großen Spannung zwischen dem Zustand der Welt und den Zielen? Wer kann Einfluss nehmen? Wer oder was steuert? Eng verknüpft mit der Frage der Beeinflussbarkeit des Systems und der maßgeblichen Kräfte ist die Frage der Planbarkeit.

Ein Schwenk auf mein Fachgebiet sei erlaubt. Bei der Analyse historischer Veränderungen von Landnutzung und Landschaften wird oft unterschieden zwischen naturräumlichen, kulturellen, ökonomischen, politischen und technologischen Triebkräften (Bürgi et al. 2004). Diese wirken zum Teil direkt, zum Teil über Akteure verschiedener institutioneller Ebenen. Veränderungen werden in der Regel durch zahlreiche Faktoren beeinflusst, dennoch kann man die wichtigsten davon benennen und ihr Zusammenwirken untersuchen. Eine solche Analyse erlaubt somit auch die Abschätzung des relativen Einflusses politischer Prozesse.

Ist die Entwicklung der Landnutzung beziehungsweise der internationalen Landwirtschaft planbar, und wenn ja, durch wen und wie? Oder ist dieses System nicht ganz wesentlich durch unvorhergesehene Entwicklungen und Brüche geprägt? Einiges spricht für Letzteres - dazu zwei Beispiele aus neuerer Zeit:

Kontakt: PD Dr. Matthias Bürgi | Eidgenössische Forschungsanstalt für Wald, Schnee und Landschaft (WSL) | Gruppe Landnutzungsgeschichte | Zürcherstr. 111 | 8903 Birmensdorf | Schweiz | Tel.: +41447392354 | E-Mail: matthias.buergi@wsl.ch 
- Noch vor wenigen Jahren hat wohl kaum jemand ernsthaft damit gerechnet, dass die rasant steigende Nachfrage nach Milch und Milchprodukten in China Auswirkungen auf die Perspektiven der europäischen Milchbauern haben könnte und so letztlich Spuren in der hiesigen - hier durchaus auch physisch gemeinten - Agrarlandschaft hinterlassen würde.

- Wer war Zyniker genug zu prophezeien, dass wir versuchen würden, die spärlichen Nahrungsmittel der global Marginalisierten und Benachteiligten durch die Motoren unserer Autos zu jagen? Nachdem wir die oberirdischen Wälder großflächig gerodet und die "unterirdischen Wälder“" (Sieferle 1982) weitgehend ausgebeutet haben, stolpern wir zurück an die Erdoberfläche und machen uns über die nächste Möglichkeit her, unseren gewohnten Lebensstil noch ein wenig weiterführen zu können.

Nun mag man einwenden, dass Planung bei derart rasanten Veränderungen gar nicht in der Lage sein kann, rechtzeitig die richtigen Instrumente zu entwickeln und adäquate Maßnahmen einzuleiten. Doch dann müssten zumindest längerfristige Entwicklungen von Landschaft und Landnutzung planbar sein. Dieser Frage ging eine Studie über die Landschaftsveränderungen im schweizerischen Limmattal von 1930 bis 2000 nach (Hersperger und Bürgi eingereicht). Es zeigte sich, dass die dokumentierten Landschaftsveränderungen nur zu rund einem Viertel durch politische und planerische Maßnahmen verursacht waren. Bei den der Entwicklung der Landwirtschaft zuzuschreibenden Veränderungen waren es sogar nur 16 Prozent. Der Einfluss naturräumlicher, kultureller, ökonomischer und technologischer Triebkräfte scheint die Planbarkeit der Landnutzung also erheblich zu relativieren.

Viele historische Beispiele für Veränderungen in der Landnutzung bietet die Agrargeschichte. Altwegg (1980) hat etwa die Entwicklung des Weinbaus am Zürichsee untersucht. Zwischen 1800 und 1880 verdoppelte sich in dieser Region die Rebfläche auf rund 2000 Hektar. Doch bereits um 1930 wuchsen nur noch auf 265 und um 1966 lediglich noch auf 86 Hektar Reben. Was waren die Gründe für Aufstieg und Fall der Rebkultur? Der Aufstieg beruhte nicht nur auf klimatisch günstigen Jahren mit guten Erträgen, sondern vor allem auch auf der Industrialisierung, die zu besseren Einkommensverhältnissen weiter Bevölkerungskreise und einer wachsenden, durstigen Arbeiterschaft führte. Zudem konnte durch den Ausbau der Infrastruktur Wein kostengünstiger transportiert und in größerem Umkreis verkauft werden. Derselbe Faktor spielte allerdings auch eine zentrale Rolle beim Rückgang der Rebflächen nach 1880, indem nämlich billigere Weine aus Südfrankreich und Spanien - nach Eröffnung des Gotthardtunnels 1882 zunehmend auch aus Italien - per Bahn in die Schweiz gebracht werden konnten. Weitere Konkurrenz erwuchs dem Zürcher Wein durch die Kunstweinproduktion (oft auf Basis getrockneter ausländischer Trauben), Weinfälschungen und das Bier. Zusätzlich machten den Weinbauern steigende Arbeits- und Produktionskosten zu schaffen: Die Industrialisierung schuf nicht nur Absatzmöglichkeiten, sondern führte zur Kon- kurrenz um Arbeitskräfte zwischen erstem und zweitem Sektor. Seit den 1880er Jahren verursachten der Falsche Mehltau (eine Pilzkrankheit) und die Reblaus (ein aus Nordamerika eingeschleppter Rebschädling) in den zürcherischen Weinbergen große Schäden. Zusammen mit einer Reihe katastrophaler Fehljahre mit Hagel, Frost, schlechten Blühbedingungen und nasskalten Sommern hatten all diese Entwicklungen zur Folge, dass viele Winzer resigniert ihre Weinberge in Äcker und Wiesen umwandelten. Dass sich seither die Rebfläche trotz enormen Baudrucks in der Nähe zur boomendem Stadt Zürich wieder auf über 100 Hektar ausgedehnt hat, ist auf Neuerungen in Pflanzenschutz und Kulturmethoden, Professionalisierung der Ausbildung, günstige klimatische Veränderungen, Förderung der Weinqualität und steigendes Interesse an regionalen Produkten zurückzuführen.

Die in diesem Beispiel relevanten Faktoren wie Technologie, Klima, Schadorganismen, Infrastruktur, Nachfrage und Qualität der Produkte sind nach wie vor und weltweit für die Entwicklung der Landwirtschaft relevant. Sind diese Faktoren planbar? Können sie durch Prozesse wie das Agassessment gesteuert werden? Teilweise sicher, umfassend jedoch kaum.

Sollen Langfriststrategien erfolgreich sein, müssen sie auf die Beeinflussung der wichtigsten Triebkräfte abzielen und möglichst resistent sein gegen fundamentale Überraschungen, gegen Unplanbares. Historische und aktuelle Beispiele zeigen, dass die internationale Landwirtschaft ausgesprochen anfällig für ungeplante Entwicklungen ist. Dies lässt mich stark an der Wirksamkeit des Agassessment zweifeln - so sehr ich hoffe, dass sich die Welt in die darin angestrebte Richtung entwickeln wird.

\section{Literatur}

Altwegg, A. M. 1980. Vom Weinbau am Zürichsee. Stäfa: Th. Gut.

Bürgi, M., A. Hersperger, N. Schneeberger. 2004. Driving forces of landscape change - current and new directions. Landscape Ecology 19: 857-868.

Hersperger, A., M. Bürgi. Eingereicht. How do policies shape landscapes? Landscape change and its political driving forces in the Limmat Valley, Switzerland, 1930-2000. Landscape and Urban Planning.

IAASTD (International Assessment of Agricultural Knowledge, Science and Technology for Development). 2008. IAASTD - Global summary for decision makers. www.agassessment.org/docs/Global_SDM_060608_English.pdf (abgerufen 25.07.2008).

Sieferle, R. P. 1982. Der unterirdische Wald: Energiekrise und industrielle Revolution. München: C. H. Beck.

Eingegangen am 14. Januar 2008; überarbeitete Fassung angenommen am 17. Juli 2008.

\section{Matthias Bürgi}

Geboren 1967 in Bern. Studium der Umweltnaturwissenschaften an der ETH Zürich. 1997 Promotion. 1998 bis

2001 Postdoktorand an der Harvard University, Cambridge, MA, und an der University of Wisconsin, Madison, WI. Anschließend Oberassistent an der Professur für Naturund Landschaftsschutz der ETH Zürich. 2006 Habilitation in Historischer Ökologie an der ETH Zürich. Seit 2006 Leiter der Gruppe Landnutzungsgeschichte an der WSL Birmensdorf. Seit 2004 Weinbauer am Zürichsee. 